\title{
T helper subset development: roles of instruction, selection, and transcription
}

\author{
J. David Farrar, Helene Asnagli, and Kenneth M. Murphy \\ Department of Pathology and Immunology, Howard Hughes Medical Institute, Washington University School of Medicine, \\ St. Louis, Missouri, USA
}

Address correspondence to: Kenneth M. Murphy, Department of Pathology and Immunology, Howard Hughes Medical Institute, Washington University School of Medicine, 660 S. Euclid Avenue, St. Louis, Missouri 63110, USA.

Phone: (314) 362-2009; Fax: (314) 747-4888; E-mail: murphy@immunology.wustl.edu.

J. Clin. Invest. 109:431-435 (2002). DOI:10.1172/JCI200215093.

The past 15 years have seen the development of a field in immunology entirely devoted to understanding the divergence of $\mathrm{CD}^{+} \mathrm{T}$ cells into distinct cytokine-producing subsets. Generally speaking, the immune response to a pathogen can develop toward a more strongly cellular type (type 1), or toward a more allergic type of response (type 2). When well developed, these gear the immune response for the effective elimination of different types of pathogens, with a type 1 response being more effective against intracellular pathogens, for example Listeria monocytogenes or mycobacteria. Type 2 allergic responses, in contrast, favor the elimination of parasites such as helminths. The basis for this selective action resides in the cytokines made by the $\mathrm{CD} 4^{+} \mathrm{T}$ helper cell subsets in these responses. T helper type 1 (Th1) cells produce several characteristic cytokines, most notably IL-2 and IFN- $\gamma$, whereas Th 2 cells produce a set of cytokines, most notably IL-4, IL-5, and IL-13. In turn, IL-2 and IFN- $\gamma$ promote the development of strong cell-mediated immunity, whereas the type 2 cytokines promote allergic responses effective in eliminating parasites.

When confronted with a pathogen, it is important that the immune system activate the appropriate type of response. Fortunately, it has developed reliable mechanisms that help naive CD4 T cells in this choice. These mechanisms have been fairly well worked out at many levels over the last decade, and several thorough reviews have described recent findings regarding the signaling pathways and transcription factors that contribute to peripheral $\mathrm{CD}^{+}$Th development (1-3).

Despite the great progress in the molecular understanding of these processes, there are still issues in this area that are controversial and actively debated. Here, we will focus on some aspects that are unresolved within the various models of Th1 and Th2 development and will try to fit together some of the recent observations that have motivated these somewhat theoretical considerations. In particular, we will take this opportunity to focus on a difficult topic, that of whether Th1/Th2 development rests on selective or instructive mechanisms, a general issue that is also debated in regard to CD4/CD8 lineage commitment and is common to many developmental systems. Distinguishing between strictly selective and instructive models is difficult, as many experimental results can be compatible with both interpretations.

\section{Cytokines and the Th1/Th2 balance}

Cytokines clearly are important in the development of different cytokine-producing Th 1 and Th 2 cells. IL-12 secreted by activated macrophages and dendritic cells promotes Th1 development with IFN- $\gamma$ secretion $(4,5)$. Type I IFNs (IFN- $\alpha$ and $-\beta$ ) also promote Th1 responses in human $\mathrm{CD}^{+} \mathrm{T}$ cells, although they do not appear to do so efficiently in the mouse (6-9). IL-4, conversely, promotes Th2 development. The source of IL-4 initiating this process is debated $(10,11)$.

There is little doubt that cytokines at least are very significant modifiers of Th development, along with other parameters of activation that can skew development, such as antigen dose or affinity (12). The cellular and molecular steps leading to the polarization in bulk populations of $\mathrm{T}$ cells, in contrast, are not well understood. Coffman and Reiner have outlined alternative mechanisms that could underlie Th1/Th2 polarization, including a selective cytokine-induced outgrowth of precommitted $\mathrm{T}$ cells, or the instructive actions of cytokines on naive uncommitted $\mathrm{T}$ cell precursors (13). Selective models were initially attractive because of evidence for cytokine-independent and Stat-independent phenotype effects $(14,15)$, as well as for apparently random, or "stochastic," aspects of cytokine gene expression. For example, in some cases, only one cytokine allele, but not both, is expressed in Th differentiation (16-18), consistent with a selective rather than instructive model. Thus, naive T cells may already be clonally committed to certain fates or may generate random mixtures of fixed fates upon their initial stimulation. In either case, the fates here are fixed, and IL-12 or IL-4 would act by selecting certain clones for cell survival and growth. These data raised 
the possibility of selection in Th1 and Th2 polarization, but they did not settle the matter, since both instructive and selective models can accommodate stochastic features of cytokine expression.

The term "stochastic" implies only that there is a probability associated with gene expression in a cell. For example, naive $\mathrm{CD} 4^{+} \mathrm{T}$ cells may have a low, but non-zero, probability of expressing IL-4, since low levels of IL-4 can be detected upon strong stimulation of even naive $T$ cells. Both the selective models can accommodate this observation. Selective models posit that this is due to the generation of multiple phenotypes in early rounds of division; instructive models argue that relatively low levels of Th2-specific transcription factors (e.g., GATA-3) inefficiently augment IL-4 transcription. Selective and instructive models are distinguished by the point at which cell fate commitment is proposed to occur, relative to the timing of the cytokine effect. In instructive models, cytokines (e.g., IL-12 or IL-4) are necessary for causing commitment rather than cell outgrowth. Cytokine signaling induces changes in gene expression, which signifies fate determination. Therefore, instructive models place cytokines before commitment. By contrast, in selective models, fate commitment precedes cytokines, which only act to alter cell outgrowth but do not change gene expression within a clonal line.

These differences appear subtle but have fundamentally different implications. Th1/Th2 polarization via instruction implies that the next questions to answer relate to the changes in transcription factors and cytokine transcription following cytokine-signaling. Polarization via selection implies we must next answer how cytokine-signaling can selectively alter cell survival and growth.

\section{The cell cycle, cell division, and cytokine gene expression}

Reiner and colleagues made the very original and interesting observation that IL-4 production occurs at increased frequencies in cells that have undergone at least three cell divisions (19). Further, they found that IFN- $\gamma$ production required at least one round of cell division. IL-4 production was blocked by inhibitors that block cells at G1/S and G2/M checkpoints, which they interpreted to indicate that at least one cell division is required for the initiation of IL-4 transcription.

Two recent reports build on this observation and suggest a somewhat different interpretation. Radbruch and colleagues determined that IL-4 induction occurs in cells that have not progressed through the first cell division upon $\mathrm{T}$ cell activation (20). Here, the length of exposure to both $\mathrm{T}$ cell receptor (TCR) activation and IL-4 signaling was important in commitment to IL-4 synthesis. Similar findings by Paul and colleagues indicate that both IFN- $\gamma$ and IL-4 are regulated by the duration of signaling through these pathways (21). These studies clearly show that in the first few cell divisions, naive $\mathrm{T}$ cells modify cytokine gene chromatin and alter cytokine expression, findings that are not inconsistent with either selective or instructive models of development. Since none of these studies traced individual cell fates, however, it has not been resolved whether cytokines alter the probability of gene expression in a clone or induce selective outgrowth of cells that have randomly committed to a specified fate.

\section{Signaling, transcription, chromatin remodeling, and fate determination}

Reasonable models of differentiation should account for known effects of signaling pathways and transcription factors on Th1/Th2 development. GATA-3 and T-bet are transcription factors highly, reciprocally induced early on in polarizing conditions. GATA-3 is selectively induced early in Th2 development (22), induced by IL- 4 through Stat 6 , and inhibited by IFN- $\gamma$ and IL-12 through Stat 1 and Stat4 (23). GATA-3 overexpression increases Th 2 cytokine expression (22) and inhibits IL-12R $\beta 2$ expression with decreased Th1 development even in Th1-inducing conditions $(23,24)$. GATA-3 induces Th2 development in Stat6-deficient $\mathrm{T}$ cells and induces autoactivation of the endogenous GATA3 gene (25).

In concordance with instructive models, Th2-specific regulatory elements have been recently identified within the Th2 cluster. First, Arai and colleagues (26) identified Th2-specific DNase hypersensitive (HS) sites between the IL4 and IL13 genes, termed HSS-1 and HSS-2, found later to be within the conserved noncoding region 1 (CNS-1 region) (27). Other HS sites were found in IL4 and IL13 genes using similar approaches but in developing Th2 $\mathrm{CD}^{+} \mathrm{T}$ cells (28), particularly the intronic enhancer (IE) located in the first IL4 intron. Ranganath et al. (29) found that GATA-3 augments enhancer activity of certain genomic regions near IL4 and IL13 in cooperation with the IL4 promoter. While these authors did not identify how or where this augmentation occurred, direct evidence that GATA- 3 acts at one or both of these sites is found in the work of Flavell and colleagues (30), who used transgenic analysis of the IL4 promoter in combinations with various regions including HSS and IE. In this study, both HSS and IE elements enhanced IL4 promoter activity, but the combination of both when used in a minilocus showed the greatest level of expression and, more importantly, Th2 specificity. Further, this minilocus was directly responsive to GATA-3 when introduced by retrovirus into T cells, suggesting that these sites may represent the places where GATA-3 acts to begin the process of general activation of the Th2 cytokine cluster for Th2-specific expression. In addition, Miyatake and colleagues showed that GATA-3 can directly interact with the CNS-1 region (31). Finally, Mohrs et al. have very recently shown that the CNS-1 region is definitely required in the native response of Th2 cytokines to the effects of IL-4 for Th2 development (32). Together, these studies paint a picture of a Th2 cytokine cluster coordinately controlled by interactions between separated enhancer-like regions developmentally responsive to the GATA-3 transcription factor. 


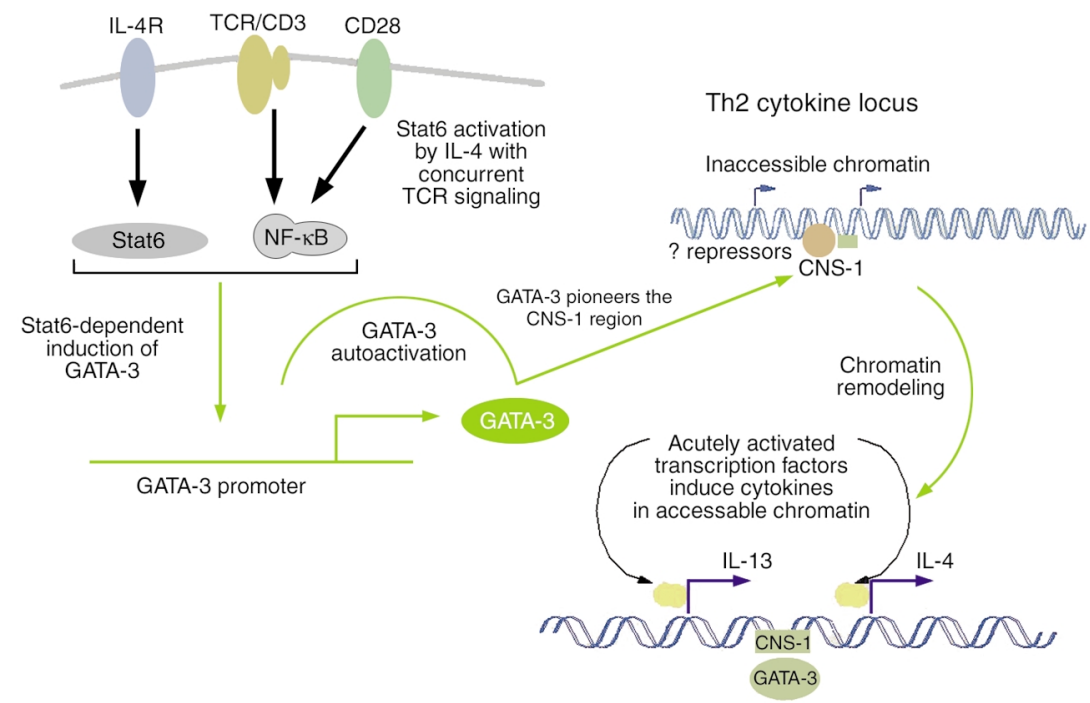

\begin{abstract}
Figure 1
Major pathways thought to regulate the development of T cells with the Th2 phenotype. Th2 cells typically promote allergic symptoms and asthma, as well as the systemic responses that help combat parasitic responses. As discussed in the text, it is still uncertain whether the effects of various factors are most consistent with an instructive or a selective route to T cell differentiation. Nevertheless, the transcription factor GATA-3 appears to be central to this response. Binding of GATA-3 to genes encoding the characteristic Th2 cytokines is an essential first step in the activation of these genes. In addition, GATA-3 autoactivates its own gene expression, thus stabilizing the Th2 phenotype through an intracellular (intrinsic) positive feedback loop.
\end{abstract}

How can these observations fit within instructive or selective models of differentiation? The above studies suggest an instructive role for GATA-3 in directing commitment to the Th2 fate and would place GATA-3 downstream of the effects of cytokine signaling. However, Stat6-independent Th2 development has been observed in vivo and in vitro (33). Since wild-type levels of GATA-3 are found in Stat6-deficient Th2 cells (25), it seems that the "instructive" effects of IL-4 signaling can be uncoupled from GATA-3 expression and Th2 development, implying a Stat6-independent stochastic process in early commitment. By this thinking, GATA-3 might induce Th2 development through a mechanism that is not "instructive," in that GATA-3 expression would not result from signals delivered by cytokines. Likewise, overexpression of GATA-3 promotes Th2 development in bulk populations, but this might be due either to instructive changes in transcriptional programs or to the selective outgrowth of cells already committed to the Th2 fate. That GATA-3 might instruct $\mathrm{Th} 2$ transcriptional programs was suggested by its ability to reprogram cytokine expression in previously differentiated cell $(34,35)$, although these experiments were not explicitly designed to track individual cell fates. This issue has been tested using retroviral techniques to tag individual $\mathrm{CD} 4^{+} \mathrm{T}$ cells and follow their fate during development. Farrar et al. (36) found that retrovirus-induced GATA-3 expression in developing clonal progenitors favors commitment to the Th2 fate even in Stat6-deficient T cells. The finding that GATA-3 expression in this context induces no apparent selective outgrowth compared with control retrovirus provides prima facie support for the instructive rather than the selective model.

\section{GATA-3 regulation through multiple pathways}

Besides being induced through Stat6, GATA-3 expression may also be regulated by other signals. For this reason, the stochastic Th2 commitment observed in the absence of IL-4 might be due to GATA-3 expression that was induced through other pathways. GATA-3 appears to activate its own transcription, either directly or indirectly $(25,37)$, and indeed, a doublet GATAbinding element residing within the first intron of GATA3 (Figure 1) is required for promoter activity in reporter assays (38). GATA-3 autoactivation may therefore act as a stabilizing influence in Th2 commitment. Second, signaling through CD28 augments GATA-3 expression (39). Also, naive $\mathrm{CD} 4^{+} \mathrm{T}$ cells require NF- $\mathrm{KB}$ p50 activation, presumably downstream of TCR signaling for GATA-3 expression (40). Consistent with

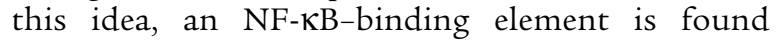
upstream of the murine GATA3 transcriptional start site (40) (Figure 1). CD28-dependent costimulation augments Th2 responses to infection with Shistosoma mansoni (41). Finally, a direct link between GATA-3 expression and CD28 signaling was recently proposed for rat $\mathrm{CD} 4^{+} \mathrm{T}$ cells (39). Thus, GATA-3 may be controlled by more than simply Stat 6 activation, and signals that elevate GATA-3 transiently above its threshold for autoactivation can apparently tip the balance toward Th2 development.

In summary, for Th2 development, there is strong evidence that IL-4 acts through Stat 6 activation to elevate GATA-3 transcription. After transient elevation beyond some threshold, GATA-3 autoactivation occurs, stabilizing its own expression and somehow activating the Th2 cytokine cluster - perhaps by interactions with sites such as CNS- 1 and IE, increasing promoter accessibility to acutely activated 
transcription factors. Because GATA-3 does not appear to act by increasing selective cell outgrowth, it can be said to exert instructive effects. Nonetheless, there may still be Stat6-dependent mechanisms unrelated to GATA-3 that exert effects on cellular outgrowth, allowing IL- 4 and Stat 6 to have combined instructive and selective effects.

\section{Mechanisms in Th1 development}

Recently, Glimcher and colleagues (42) found a novel transcription factor, T-bet, a member of the T-box family of transcription factors that is restricted to Th1 cells and promotes Th1 development. Initial functional characterization showed that T-bet is expressed within 24 hours of primary activation under Th1-inducing conditions. Retroviral overexpression of T-bet in naive $\mathrm{CD}^{+} \mathrm{T}$ cells increases IFN- $\gamma$ production and inhibits Th 2 cytokines, even in cells cultured under Th2-inducing conditions. In addition, T-bet appears to regulate the IFN- $\gamma$ promoter, suggesting that it directly induces Th1 fate (42), and may provide the connection between the signal delivered by IL-12 and Stat 4 and the induction of IFN- $\gamma$ by Th 1 cells.

Although Stat4-independent Th1 development in CD4 ${ }^{+}$cells has been reported $(15,43)$, it has been seen only in the abnormal setting of double deficiency for both Stat 6 and Stat 4 , and there is significant evidence that Stat 4 activation is required for IFN- $\gamma$ production in vivo $(44,45)$, particularly in $\mathrm{CD}^{+} \mathrm{T}$ cells (14). In $\mathrm{CD}^{+} \mathrm{T}$ cells, IFN- $\gamma$ production can occur without Stat4 (14). Potentially, the use of various modes of stimulation (TCR, cytokines, or chemical activation) may involve different requirements for Stat 4 in IFN- $\gamma$ production or Th1 development.

The initial T-bet study suggested that IL-12 induces T-bet through Stat 4 activation, providing the instructive link between IL-12 and Th1 development. However, a more recent study (46) shows that T-bet is expressed at normal levels in Stat4-deficient T cells. Importantly, IL-4 inhibits T-bet expression, whereas retroviral T-bet expression can induce IL-12R $\beta 2$ and Th1 commitment. These results seem to place T-bet not downstream of IL-12 and Stat4, but upstream. Finally, T-bet-expressing cells display increased proliferation, suggesting potentially a selective outgrowth model for T-bet-induced Th1 commitment. The strong early induction of T-bet in Th1-inducing conditions, coupled with the normal expression in Stat4-deficient $T$ cells, suggests that there may be another pathway controlling its expression, and indeed, very recent work indicates that T-bet is actually controlled by Stat1, rather than Stat4 (47). To distinguish selective from instructive models in T-bet-dependent Th1 development would require direct cell fate tracking, as has been carried out for Th2 development (36).

\section{Conclusions}

This review has focused on a limited range of issues within the larger topic of Th1/Th2 regulation. It is important to note here that selective and instructive models of differentiation are not mutually exclusive.
Clearly, both IL-12 and IL-4 provide proliferative signals to a variety of cell types. Further, signals delivered through Stat 4 and Stat 6 can serve dual functions of commitment and selective outgrowth. Defining their function in these processes is the challenge left in this field.

Th1/Th2 regulation is exceedingly complex, but its importance is unquestionable, particularly in the study of such diverse diseases as allergies and asthma, as well as type 1 diabetes and other autoimmune disorders. This is an active area of research for the design of immunomodulatory therapies intended either to dampen overreactive responses or to strengthen weak ones. Magic bullets and master switches may be rare commodities in this area. Nonetheless, defining all of the mechanisms controlling these processes, including the ones discussed in this review, will help make rational therapies that manipulate Th1/Th2 balance a reality.

1. Murphy, K.M., et al. 2000. Signaling and transcription in T helper development. Annu. Rev. Immunol. 18:451-494.

2. Kuo, C.T., and Leiden, J.M. 1999. Transcriptional regulation of T lymphocyte development and function. Annu. Rev. Immunol. 17:149-187.

3. Glimcher, L.H., and Murphy, K.M. 2000. Lineage commitment in the immune system: the $\mathrm{T}$ helper lymphocyte grows up. Genes Dev. 14:1693-1711.

4. Hsieh, C.S., et al. 1993. Development of THI CD4+ T cells through IL-12 produced by Listeria-induced macrophages. Science. 260:547-549.

5. Macatonia, S.E., et al. 1995. Dendritic cells produce IL-12 and direct the development of Th1 cells from naive CD4+ T cells. J. Immunol. 154:5071-5079.

6. Rogge, L., et al. 1998. The role of Stat 4 in species-specific regulation of Th cell development by type I IFNs. J. Immunol. 161:6567-6574.

7. Cho, S.S., et al. 1996. Activation of STAT4 by IL-12 and IFN-alpha: evidence for the involvement of ligand-induced tyrosine and serine phosphorylation. J. Immunol. 157:4781-4789.

8. Farrar, J.D., Smith, J.D., Murphy, T.L., and Murphy, K.M. 2000. Recruitment of Stat 4 to the human interferon-alpha/beta receptor requires activated Stat2. J. Biol. Chem. 275:2693-2697.

9. Farrar, J.D., et al. 2000. Selective loss of type I interferon-induced STAT4 activation caused by a minisatellite insertion in mouse STAT2. Nat. Immunol. 1:65-69.

10. Seder, R.A., Paul, W.E., Davis, M.M., and Fazekas, D.S. 1992. The presence of interleukin 4 during in vitro priming determines the lymphokine-producing potential of CD4+ T cells from $\mathrm{T}$ cell receptor transgenic mice. J. Exp. Med. 176:1091-1098.

11. Swain, S.L., Weinberg, A.D., English, M., and Huston, G. 1990. IL-4 directs the development of Th2-like helper effectors. J. Immunol. 145:3796-3806

12. Constant, S.L., and Bottomly, K. 1997. Induction of Th1 and Th2 CD4+ $\mathrm{T}$ cell responses: the alternative approaches. Annu. Rev. Immunol. 15:297-322.

13. Coffman, R.L., and Reiner, S.L. 1999. Instruction, selection, or tampering with the odds? Science. 284:1283.

14. Carter, L.L., and Murphy, K.M. 1999. Lineage-specific requirement for signal transducer and activator of transcription (Stat) 4 in interferon gamma production from CD4(+) versus CD8(+) T cells. J. Exp. Med. 189:1355-1360.

15. Kaplan, M.H., Wurster, A.L., Smiley, S.T., and Grusby, M.J. 1999. Stat6dependent and -independent pathways for IL-4 production. J. Immunol. 163:6536-6540.

16. Hollander, G.A., et al. 1998. Monoallelic expression of the interleukin-2 locus. Science. 279:2118-2121.

17. Riviere, I., Sunshine, M.J., and Littman, D.R. 1998. Regulation of IL-4 expression by activation of individual alleles. Immunity. 9:217-228.

18. Naramura, M., Hu, R.J., and Gu, H. 1998. Mice with a fluorescent marker for interleukin 2 gene activation. Immunity. 9:209-216.

19. Bird, J.J., et al. 1998. Helper T cell differentiation is controlled by the cell cycle. Immunity. 9:229-237.

20. Richter, A., Lohning, M., and Radbruch, A. 1999. Instruction for cytokine expression in $\mathrm{T}$ helper lymphocytes in relation to proliferation and cell cycle progression. J. Exp. Med. 190:1439-1450.

21. Ben-Sasson, S.Z., Gerstel, R., Hu-Li, J., and Paul, W.E. 2001. Cell division is not a "clock" measuring acquisition of competence to produce IFN-gamma or IL-4. J. Immunol. 166:112-120. 
22. Zheng, W., and Flavell, R.A. 1997. The transcription factor GATA-3 is necessary and sufficient for Th2 cytokine gene expression in CD4 T cells. Cell. 89:587-596.

23. Ouyang, W., et al. 1998. Inhibition of Th1 development mediated by GATA-3 through an IL-4-independent mechanism. Immunity. 9:745-755.

24. Ferber, I.A., et al. 1999. GATA-3 significantly downregulates IFN-gamma production from developing Th1 cells in addition to inducing IL-4 and IL-5 levels. Clin. Immunol. 91:134-144.

25. Ouyang, W., et al. 2000. Stat6-independent GATA-3 autoactivation directs IL-4-independent Th2 development and commitment. Immunity. 12:27-37.

26. Takemoto, N., et al. 1998. Th2-specific DNase I-hypersensitive sites in the murine IL-13 and IL-4 intergenic region. Int. Immunol. 10:1981-1985.

27. Loots, G.G., et al. 2000. Identification of a coordinate regulator of interleukins 4,13 , and 5 by cross-species sequence comparisons. Science. 288:136-140.

28. Agarwal, S., and Rao, A. 1998. Modulation of chromatin structure regulates cytokine gene expression during $\mathrm{T}$ cell differentiation. Immunity. 9:765-775.

29. Ranganath, S., et al. 1998. GATA-3-dependent enhancer activity in IL-4 gene regulation. J. Immunol. 161:3822-3826.

30. Lee, G.R., Fields, P.E., and Flavell, R.A. 2001. Regulation of IL-4 gene expression by distal regulatory elements and GATA-3 at the chromatin level. Immunity. 14:447-459.

31. Takemoto, N., et al. 2000. Cutting edge: chromatin remodeling at the IL-4/IL-13 intergenic regulatory region for Th2-specific cytokine gene cluster. J. Immunol. 165:6687-6691.

32. Mohrs, M., et al. 2001. Deletion of a coordinate regulator of type 2 cytokine expression in mice. Nat. Immunol. 2:842-847.

33. Jankovic, D., et al. 2000. Single cell analysis reveals that IL-4 receptor/Stat 6 signaling is not required for the in vivo or in vitro development of CD4(+) lymphocytes with a Th2 cytokine profile. J. Immunol. 164:3047-3055

34. Kurata, H., Lee, H.J., O'Garra, A., and Arai, N. 1999. Ectopic expression of activated Stat 6 induces the expression of Th2-specific cytokines and transcription factors in developing Th1 cells. Immunity. 11:677-688.

35. Lee, H.J., et al. 2000. GATA-3 induces T helper cell type 2 (Th2) cytokine expression and chromatin remodeling in committed Th1 cells. J. Exp. Med. 192:105-115.

36. Farrar, J.D., et al. 2001. An instructive component in T helper cell type 2 (Th2) development mediated by GATA-3. J. Exp. Med. 193:643-650.

37. Ranganath, S., and Murphy, K.M. 2001. Structure and specificity of GATA proteins in Th2 development. Mol. Cell. Biol. 21:2716-2725.

38. George, K.M., et al. 1994. Embryonic expression and cloning of the murine DATA-3 gene. Development. 120:2673-2686.

39. Rodriguez-Palmero, M., Hara, T., Thumbs, A., and Hunig, T. 1999. Triggering of T cell proliferation through CD28 induces GATA-3 and promotes $\mathrm{T}$ helper type 2 differentiation in vitro and in vivo. Eur. J. Immunol. 29:3914-3924

40. Das, J., et al. 2001. A critical role for NF-kappa B in GATA3 expression and TH2 differentiation in allergic airway inflammation. Nat. Immunol. 2:45-50.

41. King, C.L., Xianli, J., June, C.H., Abe, R., and Lee, K.P. 1996. CD28-deficient mice generate an impaired Th2 response to Schistosoma mansoni infection. Eur. J. Immunol. 26:2448-2455.

42. Szabo, S.J., et al. 2000. A novel transcription factor, T-bet, directs Th1 lineage commitment. Cell. 100:655-669.

43. Kaplan, M.H., Wurster, A.L., and Grusby, M.J. 1998. A signal transducer and activator of transcription (Stat)4-independent pathway for the development of T helper type 1 cells. J. Exp. Med. 188:1191-1196.

44. Kaplan, M.H., Sun, Y.L., Hoey, T., and Grusby, M.J. 1996. Impaired IL-12 responses and enhanced development of Th2 cells in Stat4-deficient mice. Nature. 382:174-177.

45. Thierfelder, W.E., et al. 1996. Requirement for Stat4 in interleukin-12mediated responses of natural killer and T cells. Nature. 382:171-174.

46. Mullen, A.C., et al. 2001. Role of T-bet in commitment of TH1 cells before IL-12-dependent selection. Science. 292:1907-1910.

47. Lighvani, A.A., et al. 2001. T-bet is rapidly induced by interferon-gamma in lymphoid and myeloid cells. Proc. Natl. Acad. Sci. USA. 98:15137-15142. 